\title{
1.4 Clinical picture and laboratory disorders in patients with COVID-19 infection and ischemic heart disease
}

Since December 2019, cases of disease related severe acute respiratory syndrome coronavirus 2 (SARS-CoV-2), now known as COVID-19 (coronavirus disease 2019), have rapidly spread from Hubei Province in China to the whole world. The World Health Organization (WHO) has officially recognised COVID-19 as a pandemic and countries worldwide are now facing huge challenges trying to prevent its further spread as well as treating the growing number of COVID-19 patients. In fact, although the majority of cases are usually self-limiting with mild symptoms such as low-grade fever and cough, the disease can be fatal [32].

The pathophysiology of COVID-19 is associated with an enhanced inflammatory response of the patient's body, which leads to damage not only to lung tissue but also to other body systems, including the cardiovascular system [33].

A multicenter study of 44,672 cases of COVID-19 showed a fivefold increase in mortality in patients with cardiovascular disease compared with no pathology $(10.5 \%$ vs. $2.3 \%$ ) [34], the presence of cardiovascular disease in history helps patients become more susceptible to the virus. A study of 5700 patients have reported hypertension (56.6\%), ischemic heart disease (IHD) (11.1\%) and congestive cardiac failure (6.9\%) as common underlying co-morbidities in confirmed COVID-19 cases [35].

Today, most researchers are inclined to believe that the predictors of an unfavorable IHD prognosis in patients with COVID-19 are inflammatory processes in the vascular wall caused by activation of the cascade of proinflammatory reactions, complement system and proinflammatory cytokines through to "cytokine storm". Myocardial inflammation and endothelial dysfunction caused by SARS-CoV-2 cause an imbalance between myocardial perfusion and its metabolic processes in patients with coronary heart disease, which contributes to the rupture of atherosclerotic plaque. It is known that the level of pro-inflammatory cytokines (IL-1, IL-6, tumor necrosis factor- $\alpha$ (TNF- $\alpha$ ) and others) can be used to assess patients at risk of adverse disease with COVID -19 with cardiovascular pathology [36]. However, in routine practice it is 
not always possible to quickly obtain the results of the study of the above markers of inflammation. However, it is known that the total effect of most pro-inflammatory cytokines leads to changes in general clinical blood testing, in particular, IL-6 affects leuko- and lymphopoiesis.

Thus, the identification of inflammatory markers as prognostic predictors of mortality in patients with COVID-19 and CVD may be useful in assessing the severity of the disease and making optimal treatment decisions.

The aim of the work - to investigate the connection between hospital mortality and markers of systemic inflammation in COVID-19 patients with IHD and to assess hematological indices under the influence of the inclusion of quercetin and arginine in complex therapy at the inpatient stage of treatment.

A retrospective analysis of 63 medical records of COVID-19 patients with IHD who were inpatient treatment from February to April 2021 was carried out. The diagnosis of coronary heart disease was verified on the basis of clinical data and results of laboratory and instrumental research methods in accordance with the recommendations of the European Society of Cardiology (ESC), the Association of Cardiologists of Ukraine, and protocols of the Ministry of Health of Ukraine. The presence of COVID-19 was established using polymerase chain reaction (PCR) to detect SARS-CoV-2 RNA in the test samples.

3 patients with incomplete survey data, 6 patients who died from non-COVID-19related diseases, and 2 patients with a history of malignancy were excluded from the clinical trials. A total of 52 COVID-19 patients with IHD were included in the study, including 30 males (57.69\%) and 22 females (42.31\%). The average age of the surveyed was $66.23 \pm 11.41$ years $(\mathrm{M} \pm \mathrm{m})$.

To achieve this goal, patients were divided into two groups: the first consisted of $40(76.92 \%)$ patients who were discharged with recovery; the second group included $12(23.08 \%)$ patients who died. Evaluation of hematological indices under the influence of inclusion in the complex therapy of metabolic drugs was determined in 25 patients of group I, divided into 2 subgroups. Subgroup I A, which consisted of 17 patients, received water-soluble quercetin (Corvitin, "Borshchahivskiy Chemical - 
Pharmaceutical Plant", and subgroup IB (8 patients) - arginine hydrochloride (Tivortin, "Yuria-Pharm"). The severity of the systemic inflammation syndrome in the selected groups of patients was assessed by the number of leukocytes and the cellular composition of peripheral blood upon admission to the hospital and, based on the data obtained; the value of hematological integral indices was calculated: 1) blood leukocytes shift index (BLSI) with N.I. Yabluchansky (1983): BLSI $=(E+B+S+$ $\mathrm{RS}+\mathrm{Y}+\mathrm{M}) /(\mathrm{Mo}+\mathrm{L})$, where E - eosinophils, B - basophils, S - segmented neutrophils, RS - rod-shaped, Y - young neutrophils, M - myelocytes, Mo - monocytes, L - lymphocytes. Normally, the BLSI indicator averages (1.96 \pm 0.17 ) conv. unit; 2) index of the neutrophil-to-lymphocyte ratio (NLR), under physiological conditions, the value of NLR is $(2.47 \pm 0.65)$ conv. unit; 3$)$ intoxication leukocyte index (ILI), with Kalf-Kalif formula: $\mathrm{ILI}=(2 \times \mathrm{RS}+\mathrm{S}) /(\mathrm{Mo}+\mathrm{L}) \times(\mathrm{E}+1)$, № (0.3-1.5) conv. unit; 4) nuclear intoxication index (NII) was calculated by GA Dashtayantsom (1978): NII = (Mo +RS) / S, normally averages (0.05-0.1) conv. unit; 5) the ratio of neutrophils to monocytes $(\mathrm{N} / \mathrm{Mo}): \mathrm{N} / \mathrm{Mo}=(\mathrm{S}+\mathrm{RS}+\mathrm{Y}) /$ Mo (Mustafina JG et al., 1999), norm $(11.83 \pm 1.31)$ conv. unit; 6$)$ the ratio lymphocyte to eosinophil (L/E) standard value $(8.73 \pm 1.26)$ conv. unit; 7$)$ the ratio of lymphocytes to monocytes (L / Mo), standard value $(5.34 \pm 0.59)$ conv. unit; 8$)$ the allergization index $(\mathrm{AI})$ : $(\mathrm{L}+10 \times(\mathrm{E}+1)) /(\mathrm{RS}$ $+\mathrm{S}+\mathrm{Mo})$, normally the indicator is (0.79-1.08) conv. unit.

The study did not include patients who underwent coronary artery bypass graft (CABG) surgery, invasive interventions with newly diagnosed coronary heart disease, cases of severe HF (functional class IV), acute cerebrovascular disease, those who died of infectious diseases other than COVID-19, and who have not reached 18 years of age.

Material processing was performed using a standard Microsoft Office Excel software package. The obtained results are presented in the form of mean value and mean error $(M \pm m)$. Student's t-test was used to determine the difference in values. Data were considered reliable under conditions $\mathrm{p}<0.05$.

According to the results of the analysis of inpatient cards, stable angina was diagnosed in $86.54 \%$ of patients, $13.46 \%$ of patients had a history of myocardial 
infarction; recurrent cases of myocardial infarction were detected in $3.84 \%$. A history of diffuse cardiosclerosis was found in $73.07 \%$ of the examined patients, postinfarction cardiosclerosis was diagnosed in $13.46 \%$ of cases.

In patients admitted to the hospital, the indicators of "red" blood were within normal range without statistical differences in the study groups.

When analysis the leukocyte of hematopoiesis, it was revealed that in patients of group II who died from cardiovascular complications associated with COVID-19, there is a significantly higher value of the number of leukocytes $(16.85 \pm 5.25) \times 10^{9} / 1$, in contrast to patients group I, who were discharged with recovery $(5.81 \pm 1.75) \times 10^{9} / 1$ $(\mathrm{p}<0.05)$. There were also statistical differences in the population of white blood cells. In particular, the number of neutrophils in group I was $(67.71 \pm 9.85) \%$, and in patients of group II $(88.09 \pm 2.99) \%$ and lymphocytes $(18.13 \pm 4.25) \%$ in group I versus $(6.25$ $\pm 1.42) \%$ in group II $(\mathrm{p}<0.05)$ (Table 1). The identified clinical features in patients of group II were accompanied by higher rates of inflammatory activity (higher total content of white blood cells and an increase in the percentage of neutrophils), with a decrease in the percentage of peripheral blood lymphocytes.

Table 1.

\section{Indicators of a general blood test in patients of the observation groups}

\begin{tabular}{|c|c|c|c|c|c|c|c|}
\hline $\begin{array}{l}\text { Clinical } \\
\text { groups }\end{array}$ & Erythrocytes & Hemoglobin & Leukocytes & $\begin{array}{c}\text { Eosinophils, } \\
\%\end{array}$ & $\begin{array}{c}\text { Neutrophils, } \\
\%\end{array}$ & $\begin{array}{c}\text { Lymphocytes, } \\
\%\end{array}$ & $\begin{array}{l}\text { Monocyt } \\
\text { es, \% }\end{array}$ \\
\hline $\begin{array}{c}\text { I } \\
\text { Group } \\
(n=40)\end{array}$ & $4.61 \pm 0.57$ & $\begin{array}{c}136.51 \pm \\
16.48\end{array}$ & $5.81 \pm 1.75$ & $2.13 \pm 1.89$ & $67.71 \pm 9.85$ & $18.13 \pm 4.25$ & $\begin{array}{c}12.03 \pm \\
4.25\end{array}$ \\
\hline $\begin{array}{c}\text { II } \\
\text { Group } \\
(n=12)\end{array}$ & $4.39 \pm 0.72$ & $\begin{array}{c}131.75 \pm \\
18.75\end{array}$ & $16.85 \pm 5.25$ & $1.69 \pm 0.91$ & $88.09 \pm 2.99$ & $6.25 \pm 1.42$ & $\begin{array}{c}6.29 \pm 1.4 \\
2\end{array}$ \\
\hline $\mathbf{p}$ & $>0.05$ & $>0.05$ & $<0.05$ & $>0.05$ & $<0.05$ & $<0.05$ & $>0.05$ \\
\hline
\end{tabular}


The analysis of integral hematological indices showed that in both groups of patients the indicators of ILI, NLR, ILI, NII were higher than normal. There was also a significantly higher level of N / Mo in patients of group II. The above indices reflect the body's systemic response to inflammation. In both groups there were below the norm of L / Mo, AI, lower levels of L / E in patients of group II, which indicates the suppression of the immune system.

The comparative analysis of hematological indices revealed significant differences, in particular, indices BLSI, N / L, N / Mo (Figure 1).

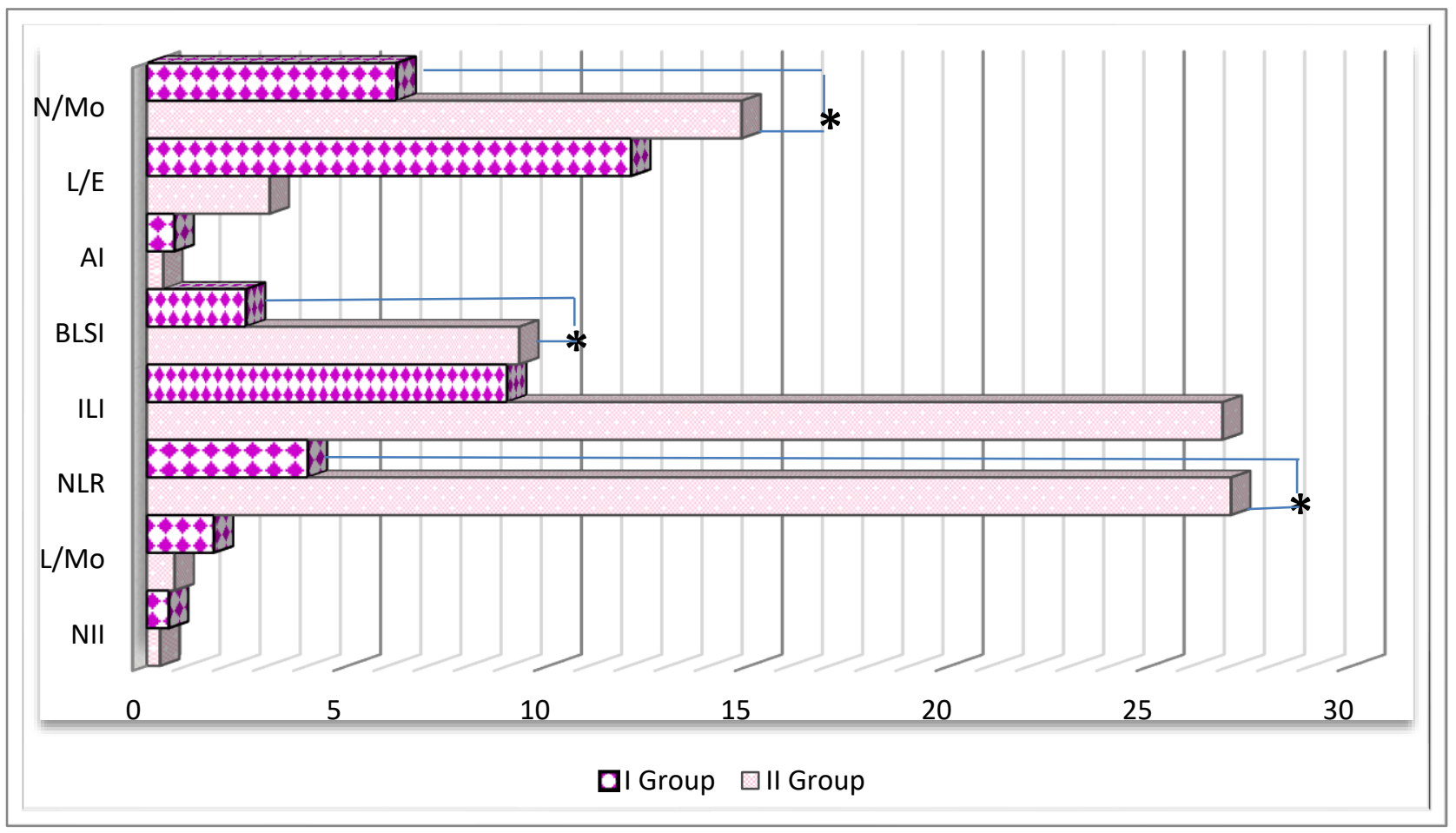

Notes: the significance of the difference between the indicators: $* \mathrm{p}<0.05$.

Figure 1. Indicators of hematological blood indices in patients of the observation groups

An increase in the BLSI index indicates an active inflammatory process and the presence of changes in immunological reactivity, which is mainly associated with an increase in the number of neutrophils and a decrease in the number of lymphocytes. A significant difference in the increase of the index BLSI in group II $(9.28 \pm 2.16)$ compared with group I $(2.47 \pm 0.95)(p<0.05)$, which is probably through to the above processes, and confirmed hemogram (Table 1). Therefore, an increase in this indicator 
can serve as an indirect criterion for an unfavorable course of the disease and its prognosis. In patients of the II clinical group, there was an increase in a number of hematological indices - N / L: $(26.99 \pm 11.11)$ versus group I $(4.01 \pm 2.36)$ and N / Mo: $(14.81 \pm 3.49)$ versus $(6.22 \pm 2.18)(\mathrm{p}<0.05)$.

The analysis of the obtained results of the distribution of hematological indices in patients of subgroup I A and I B on inpatient treatment showed that there is no significant difference between the indicators (Table 2).

Table 2.

\section{Features of distribution of hematological indices at patients on}

COVID-19 from IHD subgroups I A and I B

\begin{tabular}{|c|c|c|c|c|c|c|c|c|}
\hline $\begin{array}{l}\text { Clinical } \\
\text { groups }\end{array}$ & N/Mo & $L / E$ & $I A$ & BLSI & $I L I$ & $N / L$ & $L / M o$ & NII \\
\hline $\begin{array}{c}\text { Subgroup } \\
\text { I A } \\
\text { (Corvitin + } \\
\text { complex } \\
\text { therapy), } \\
\mathbf{n}=17\end{array}$ & $\begin{array}{c}7.33 \pm 3 . \\
83\end{array}$ & $\begin{array}{c}22.82 \pm 9 \\
.89\end{array}$ & $0.59 \pm 0.21$ & $2.15 \pm 0.86$ & $8.18 \pm 3.48$ & $3.11 \pm 1.28$ & $\begin{array}{c}2.54 \pm 1 . \\
07\end{array}$ & $\begin{array}{l}0.28 \pm 0.0 \\
8\end{array}$ \\
\hline $\begin{array}{c}\text { Subgroup } \\
\text { IB } \\
\text { (Tivortin + } \\
\text { complex } \\
\text { therapy), } \\
\mathbf{n}=\mathbf{8}\end{array}$ & $\begin{array}{c}6.31 \pm 1 . \\
22\end{array}$ & $\begin{array}{c}19.62 \pm 8 \\
.97\end{array}$ & $0.55 \pm 0.08$ & $2.03 \pm 0.48$ & $7.63 \pm 2.52$ & $2.96 \pm 0.79$ & $\begin{array}{c}2.18 \pm 0 . \\
41\end{array}$ & $\begin{array}{c}0.28 \pm 0.0 \\
6\end{array}$ \\
\hline
\end{tabular}

The rationale for the use $\mathrm{N} / \mathrm{L}$ and $\mathrm{N} / \mathrm{Mo}$ to identify patients with severe COVID-19 and mortality is likely to be further explained by the different roles of neutrophils, lymphocytes and monocytes in the immune response. Neutrophils are considered to be one of the most important immune cells in protecting the airway epithelium from SARS-CoV-2 infection by locally stimulating the production of IL$1 \beta$, IL-6, TNF- $\alpha$ and reactive oxygen species. Paradoxically, neutrophil hyperactivation and recruitment intensify the acute inflammatory response and worsen epithelial tissue damage thus leading to disease progression. On the contrary, lymphocytes are very 
important leukocytes in charge of mediating immune tolerance to self-antigens, activation of pathogen-specific adaptive immunity, and, last but not less important, orchestration of immunomodulatory mechanisms [37]. Lymphocytes, including $\mathrm{T}$ and B cells, are able to orchestrate immunomodulatory mechanisms via IL-10 and transforming growth factor-beta 1 (TGF-beta 1) production, a couple of cytokines with key anti-inflammatory and wound-healing actions. In parallel, monocytes are circulating leukocytes that in humans can be sorted into three subpopulations based on the cell surface expression of the cluster of differentiation (CD) 14 and CD16. The classical monocyte subpopulation expresses high CD14 levels with no CD16 expression. Intermediate monocytes show CD14 and CD16 expression; in contrast, non-classical monocytes express very low CD14 levels accompanied by CD16 expression. Intermediate and non-classical monocytes have been shown to produce high levels of IL-1 beta in response to lipopolysaccharide and in patients with metabolic syndrome [38]. Interestingly, reduction in both intermediate and nonclassical monocyte subpopulations has been recently associated with increased severity of the SARS-CoV-2 infection [39]. Additionally, monocytes can differentiate into alternatively activated macrophages and prevent inflammatory responses by promoting tissue repair via IL-10 and TGF-beta 1 production, which may also play a pivotal role in regulating hyperactivation of the inflammatory response described in patients with severe Covid-19 [40].

Thus, it can be assumed that $\mathrm{N} / \mathrm{L}$ and $\mathrm{N} /$ Mo may reflect an imbalance between these immune cells, which in turn is associated with excessive inflammation, severe course, and mortality in group II patients. In addition, in the presence of CVD, acute inflammatory reactions can lead to ischemia. During a systemic inflammatory response, it is observed that inflammatory activity is aggravated in the coronary atherosclerotic plaque, making them more susceptible to rupture. An occlusive thrombus may be formed over a ruptured coronary plaque, caused by inflammation leading to endothelial dysfunction and elevated procoagulant activity of blood and hence it is safe to hypothesize that preexisting cardiovascular disease, in conjunction 
with an aggravated inflammatory response may result in cardiac injury, in patients that are infected with SARS-CoV-2 [36].

L-arginine, hereinafter referred to as arginine, is a semi-essential or conditionally essential amino acid, since it can be synthetized by healthy individuals but not by preterm infants. From a chemical point of view, arginine is a 2-amino-5guanidinopentanoic acid. Arginine is involved in a number of biological processes, it is the substrate for a series of reactions leading to the synthesis of other amino acids, and it is a substrate for two enzymes, namely nitric oxide (NO) synthase (NOS) and arginase, which are fundamental for the generation of NO and urea, respectively. Arginine is known to act as a substrate for NO production by endothelial cells, thus regulating vascular tone and, overall, cardiovascular homeostasis [41] and also prevents the activation and adhesion of lymphocytes and platelets [42]. This substance has antihypoxic, membrane-stabilizing, antioxidant, cytoprotective, antiradical, detoxifying effects [43]. In one study in April 2020, scientists demonstrated that the systemic manifestations observed in COVID-19 may be to endothelial dysfunction and immune inflammation [41].

Therefore, given the positive effect of arginine on endothelial function, it can be assumed that the addition of arginine may be useful in preventing endothelial dysfunction in patients with COVID-19.

Quercetin (3, 3', 4'5, 7 -pentahydroxyflavone) is a widely distributed plant flavonoid, found in several vegetables, leaves, seeds, and grains, where it is conjugated with residual sugars to form quercetin glycosides. Studies suggest that quercetin supplementation may promote antioxidant, anti-inflammatory, antiviral, and immunoprotective effects. Quercetin has been studied in various types and models of viral infection through to its promising antiviral effects in inhibiting polymerases, proteases, reverse transcriptase, suppressing DNA gyrase, and binding viral capsid proteins [44].

The drug also has an immunomodulatory effect, which consists in the suppression of the main proinflammatory cytokines TNF- $\alpha$, IL- 6 and IL- $1 \beta$ under 
conditions of "cytokine storm". The drug is a direct antioxidant, prevents cell apoptosis, and has a cardioprotective effect in ischemic and reperfusion heart disease [45, 46].

Quercetin may be useful in patients with COVID-19 with coronary heart disease through to its immunomodulatory effects and inhibition of inflammatory mediators [46], which may help reduce the "cytokine storm".

Thus, the results of our study not only suggest new markers of mortality from severe COVID-19, but also identify potential treatments aimed at reducing neutrophil hyperactivation and increasing the number of lymphocytes and monocytes.

We can draw conclusions:

1. Patients who died from cardiovascular complications associated with COVID19 had a significantly higher level of systemic inflammatory response, which is manifested by a significant increase in total white blood cell count and an increase in the percentage of neutrophils with significantly higher integrated hematological indices: BLSI, N / L and N / Mo, which is at least partially associated with the risk of disease progression to a critical condition, hospitalization in the intensive care unit and the high mortality rate of such patients.

2. Lymphopenia, excessive activation of the inflammatory cascade and heart disease - all these are the most important features of the disease COVID-19 and have high prognostic value.

3. Drugs containing arginine hydrochloride and quercetin are promising drugs in the complex therapy for the treatment of patients with COVID-19 with IHD. 